\title{
Application of Android-based Ear Biometrics Identification
}

\author{
Ni Kadek Ayu Wirdiani \\ Lecturer Department of \\ Information Technology \\ Udayana University, Bali, \\ Indonesia
}

\author{
Ni Nyoman Triana Anggra \\ Emi \\ Students Department of \\ Information Technology \\ Udayana University, Bali, \\ Indonesia
}

\author{
A. A. K. Oka Sudana \\ Lecturer in the Department of \\ Information Technology \\ Udayana University, Bali, \\ Indonesia
}

\begin{abstract}
A biometrics-based security system is a security system that uses self-recognition technology using human body parts or behavior. This study uses the ear as a feature of biometrics. The image was taken or captured by using smartphone camera; the image was converted to grayscale and Gaussian Filter to reduce noise in the image. The extraction process done by three methods, they are: Canny Edge Detection, Hough Transform, and Oriented FAST and Rotated BRIEF (ORB). Canny Edge Detection is used to get the line in the ear, Hough Transform is used to look for ear circle shape. Invariant Moments to take the image value feature for both methods. ORB is used to search the ear keypoint, to get the feature value done by taking the ORB Feature. Identification process using Euclidean Distance for Canny Edge Detection and Hough Transform, meanwhile for ORB Method used Hamming Distance calculation. Combining these three methods resulted in a match rate of $54 \%$.
\end{abstract}

\section{Keywords}

Ear Biometrics, Canny Edge Detection, Hough Transform, Oriented FAST and Rotated BRIEF, Euclidean Distance.

\section{INTRODUCTION}

The rapid developments in technology provide unlimited convenience and access to anyone, but along with rapid developments, it also provides opportunities to commit crimes through security gaps and take advantages in wrong way. Security system in digital world was created to solve the problem. Security systems such as password or Personal Identification Number (PIN) and token-based methods are particularly vulnerable to fraud because PIN number can easily forgotten or hacked and tokens can be lost, duplicated, or stolen [1]. Biometrics system can be used to solve the problems. Biometrics is a self-recognition technology using human body parts or behavior [2]. The selection requirements of biometrics are not done arbitrarily but also pay attention to several factors, such as the universal nature in which all people have it, can be distinguished from each other, are permanent thus it does not change in the long time span, easily captured, hard to be falsified, and acceptable to the general public [3].

Several biometrics development solutions have been developed to solve the problems of using conventional security. A study entitled "Online Verification System using Biometrics Face" indicates that biometrics can be applied and used as online-based verification systems [4]. Biometrics technology can be used to find or recognize authentication patterns using different features, for example by using fingerprints, iris, face shape, ear shape, signature, palm shape, palm line, walking, keystroke and sound [5]. Ear-based biometrics is not affected by environmental factors such as: mood, health, and clothing. The appearance of the ear is only affected by aging, thus, it is more suitable for long-term identification [6].

A study entitled "Development of Multimodal Identification System using Face and Ear" provides a new approach to the identification system by using biometrics face and ear of human automatically. This approach consists of three stages such as pre-processing, feature extraction with three approaches, with combined Principal Component Analysis (PCA), Linear Discriminant Analysis (LDA), and Fractal Dimensions, matching, and decision-making with regulated threshold result shows that the face and ear match are combined as biometrics [7]. Another ear biometrics study entitled "Implementation of Hough Method and Mahalanois Distance on Ear Recognition Biometric System using OpenCV Library" using Hough Method at segmentation stage and Mahalanobis Method used in image identification stage. The implementation of this system uses the OpenCV programming language and as a research object uses ear images obtained partly from data on the internet and partly obtained by using smartphone camera and high resolution digital camera [8].

Several studies have described previously used special tools or sensors to get the characteristics of ear biometrics. The solution given in this research is to make a biometrics application with smartphone as an identification tool. Smartphone was selected as application technology because they are easy to use and can be used anywhere and anytime. This is new solution and has never been given by previous research, this application titled "Application of Android-based Ear Biometrics Identification". This application uses three methods in the extraction process of ear features that is using Canny Edge Detection, Hough Transform, and Oriented FAST and Rotated BRIEF (ORB). These three methods will be used to look for ear features. Canny Edge Detection is used to find the edges or ear lines to obtain ear shape, after getting the line will be searched ear circular lines using Hough Transform, and finally Oriented FAST and Rotated BRIEF (ORB) is used to find the keypoint from the ear. This application expected to be able to recognize the ear image in accordance with the owner's data precisely and accurately.

\section{METHOD}

\subsection{Ear Biometrics}

The ear is the human organ that serves as the sense of hearing and maintaining balance [9]. The ear has an audible sound frequency range, which the frequency range is $20 \mathrm{~Hz}-20,000$ $\mathrm{Hz}$. The ear divided into the outer, middle, and inner ears. The outer ear itself is divided into earlobes, ear canal, and eardrum [10]. 
The ear shape and bone structure of pinna cartilage tissue on each person is different. The biometrics identification through the ear based on matching the distance between the pinna and the center of the earlobe. The ear has a more stable structure and does not change over time compared to the face. The ear shape is also unaffected by facial expressions. [11]. There are several methods used in ear recognition or identification systems, in general all methods are almost identical, originally ear images obtained through digital camera or other means, then converted to grayscale, after that important areas are selected and matched to existing data [12].

Iannarelli undertook ear recognition studies, first by comparing more than 10,000 ears drawn from random selecting samples in California, and a second study of fraternal and identical twins, of which physiological features are known to be similar [13]. The evidence from this study supports the hypothesis that the ear contains unique physiological features because in both studies all of the ears are examined uniquely. Identical twins have similar ears, but are not identical especially to ear structures in Concha and regional lobes. Fig. 1 shows the anatomy of the ear [14].

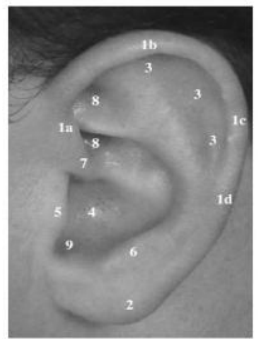

Fig 1: Helix Rim, 2 Lobule, 3 Antihelix, 4 Concha, 5 Tragus, 6 Antitragus, 7 Crus of Helix, 8 Triangular Fossa, 9 Incisure Intertragica

Iannarelli's medical research states that first ear growth after four months of birth is proportional. It turns out that although ear growth is proportional, gravity can cause the ear stretchs in a vertical direction. The effects of stretching are most prominent in the ear lobe, and from measurements indicate that the changes are non-linear. The stretch rate is about five times greater than normal during the four to eight months period. The main disadvantage of ear biometrics is that the ear can be covered by hair [15].

\subsection{Canny Edge Detection}

This algorithm provides a low error rate, localizes the edge points, and gives only one response to one edge [16]. The process for performing the Canny Edge Detection, first is to remove the noise present in the image by applying the Gaussian Filter. This process produces an image that looks a bit blurry. It aims to get the actual edge of the image, thus, the straight lines are not detected as edge [17].

Table 1. Gaussian Filter

\begin{tabular}{c|c|c|c|c|c|}
\hline \multirow{4}{*}{1} & 2 & 4 & 5 & 4 & 2 \\
\cline { 2 - 6 } & 4 & 9 & 12 & 9 & 4 \\
\cline { 2 - 6 } & 5 & 12 & 15 & 12 & 5 \\
\cline { 2 - 6 } & 4 & 9 & 12 & 9 & 4 \\
\hline & 2 & 4 & 5 & 4 & 2 \\
\hline
\end{tabular}

Subsequently, perform edge detection with one edge detection operator (Sobel or Prewitt operator) by searching horizontally $\left(\mathrm{G}_{x}\right)$ and vertically $\left(\mathrm{G}_{y}\right)$. The Sobel operator is a pair of $3 \times 3$ matrix kernels to detect vertical and horizontal edges [17].
Table 2. Sobel Operator

\begin{tabular}{|c|c|c|}
\hline+1 & +2 & +1 \\
\hline 0 & 0 & 0 \\
\hline-1 & -2 & -1 \\
\hline \multicolumn{3}{|c}{$\mathrm{Gx}$} \\
\hline
\end{tabular}

\begin{tabular}{|c|c|c|}
\hline-1 & 0 & +1 \\
\hline-2 & 0 & +2 \\
\hline-1 & 0 & +1 \\
\hline \multicolumn{3}{|c|}{ Gy }
\end{tabular}

The horizontal and vertical combined gradient spacing can be calculated using the following equation [17].

$$
|G|=\sqrt{G x^{2}+G y^{2}}
$$

Then, to calculate the resulting edge line direction, the following equation is used [17]

$$
\theta=\arctan \left(\frac{G y}{G x}\right)
$$

The next step is to divide the existing line into four separate colors with the angle and the provision such as the following [17].

a. Degrees from 0 to 22.5 and 157.5 to 180 is yellow.

b. Degree of 22.5 to 67.5 is green.

c. Degree of 67,5 to 157,5 is red.

Then minimize each edge to be slight (non-maximum surpression). The final step is to perform the binary process based on the low and high threshold values given [17].

\subsection{Hough Transform}

The idea of a Hough Transform is to create the equations of a pixel and consider all the pairs that satisfy this equation. All pairs are placed on an accumulator array, called as the transformation array [18].

Hough Circle Transform is used when a search object is in the form of a circle. The procedure used in detecting a circle is equal to the Hough Transform on the line but is done on more complex dimensional space, as in the $3 \mathrm{D}$ space parameter $\left(x_{0}, y_{0}, r\right)[17]$.

The Hough Circle Transform process includes three basic parts. The first part is edge detection. The purpose of edge detection is to decrease the number of points in the search space for the object. When the edge point is found by the edge detector, the Hough Transform algorithm is performed only at that point. The goal is to maximize signal at noise ratio and localization also minimize errors in the edge detection [17]

\subsection{Oriented FAST and Rotated BRIEF (ORB)}

ORB is basically a combination of FAST keypoint detector and BRIEF descriptor with many modifications to improve performance. First, this method uses FAST to find the keypoints, and then apply the angle size of Harris to find the best $\mathrm{N}$ points among the keypoints. FAST does not produce angular size but has a great response to the edge. Harris corner is used to measure the keypoints used. Each target number $\mathrm{N}$ for the keypoints is set with a fairly low threshold to get more than $\mathrm{N}$ keypoints, then matches Harris's size and takes the top of $\mathrm{N}$ points. FAST does not produce multi-scale features, hence pyramid scales are used on images and produce FAST (based on Harris filter) features at each pyramid level. The orientation of keypoint uses a technique known as intensity centroid [19]. 


\section{SYSTEM DESIGN}

Application of Android-based Ear Biometrics Identification consists of two general processes, they are: registration process and identification process.

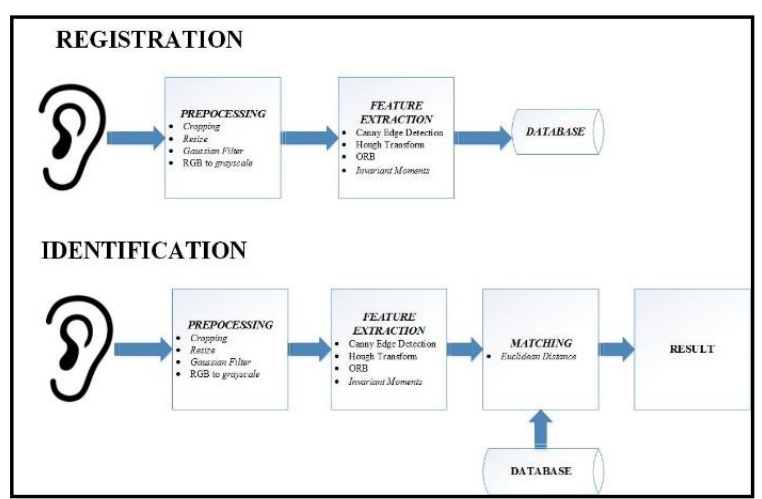

Fig 2: Design System

In general, the stages in the Application of Android-based Ear Biometrics Identification consist of the phase of image acquisition, preprocessing, matching, and determination of results [20].

\subsection{Image aqcuisition}

Phase of image acquisition or image capture done in making the application is to use the rear camera that has been available on the smartphone or through the gallery. Image captured by using camera done with a distance of $40 \mathrm{~cm}$ perpendicular from the ear. The image is automatically also saved to the photo gallery on the smartphone.

\subsection{Preprocessing}

Pre-processing stage will be done cropping process to mark Region of Interest (ROI) as the data to be examined, and then the image is converted into a smaller size (resize) with a size of 150 x150 pixel. The image then converted into grayscale image which is gray color image with color level between 0 to 255. The image that has been successfully converted to grayscale will enter the smoothing stage with the use of Gaussian Blur method. Gaussian filter is a Low Pass Filter which serves to take low frequency and remove high frequency. This filter is used to perform the process of blur effect and noise reduction. The purpose of this process is to minimize noise contained in the image thus the feature extraction will not be disturbed by other objects that are around the object image used.

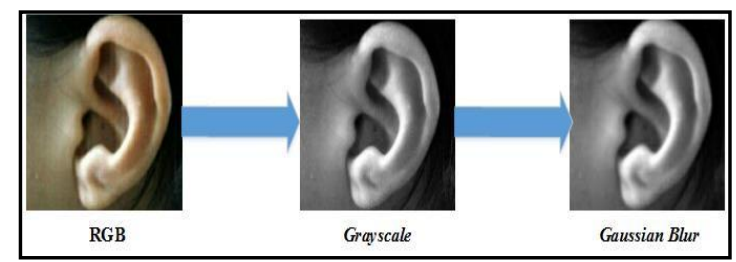

Fig 3: Proses Pre-processing

Fig 3 shows the result of pre-processing process. RGB image on the leftmost image, then converted to grayscale image thus the image becomes gray, and the rightmost image as result of the Gaussian Blur Method causes the image to look slightly blurred.

\subsection{Feature Extraction}

The feature extraction stage is a step in transforming the image into the shape of the number used as a feature of the image. The extraction process is carried out by three methods: Canny Edge Detection, Hough Transform, and ORB Method. Canny Edge Detection aims to change the image so that the edge of the image that has a high value difference. This process shows the shape and line of the ear. Hough transform is used to isolate an object by finding its limits. The Hough transform looks for a circular object in the ear. The ORB method is used to detect the keypoint. Keypoint detection is a process that uses the OpenCV Library unction to detect certain angles in the image by involving the histogram of the gradient calculation.

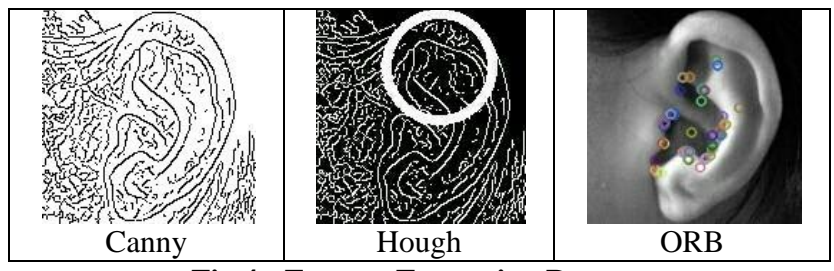

Fig 4: Feature Extraction Process

Fig 4 is a display of the results of feature extraction process. The leftmost image shows the result of Canny Edge Detection thus the lines appear on the ear, and then the middle image is the result of Hough Trasform thus the ear is obtained in the form of a circle, and the rightmost result of the ORB method to obtain keypoint on the ear. Invariant Moments method is a method that will be used in the feature extraction process with the aim to obtain the seven moment feature of an image. The advantage of the moment is unaffected by the rotation and mirror of the ear image obtained. The value of the obtained moment is stored in an array variable, whereas the result of feature extraction using the ORB method is simply taken its feature value in the form of mat. The value of the feature will be stored in the database.

\subsection{Matching}

Identification stage done on Application of Android-based Ear Biometrics Identification is by using Euclidean Distance to compare the feature value by using Invariant Moments. Euclidean Distance method will compare the vector values obtained in the three processes with the value of the image vector in the database. The ORB feature value is compared to the Hamming Distance by determining the shortest distance between the test image and the reference image.

\subsection{Result}

The decision-making stage is the last process, in this process will be decided in accordance with the results of the matching process. This process compares the value of the distance obtained at the recognition stage with the threshold, if the value obtained in accordance with the threshold range then the input data will be considered equal to the data contained with the database, otherwise, if the data does not match the threshold value then the insert data is considered not equal to the Data contained in the database, if the data obtained is same as the data contained in the database, then the application will show the identity of the owner of the data.

\section{FEATURES}

Application of Android-based Ear Biometrics Identification consists of two main features, they are: registration and recognition features.

\subsection{Registration Features}

The registration feature of the Application of Android-based Ear Biometrics Identification is a feature to perform the ear 
registration process. The registered ears are the right and left ear.

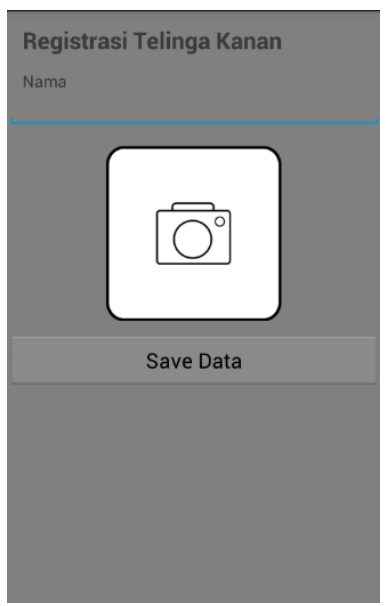

Fig 5: Registration feature

Fig 5 is the initial view to register. The first registration made is the registration of the right ear. Name data of the ear owner and ear image must be filled in to register.

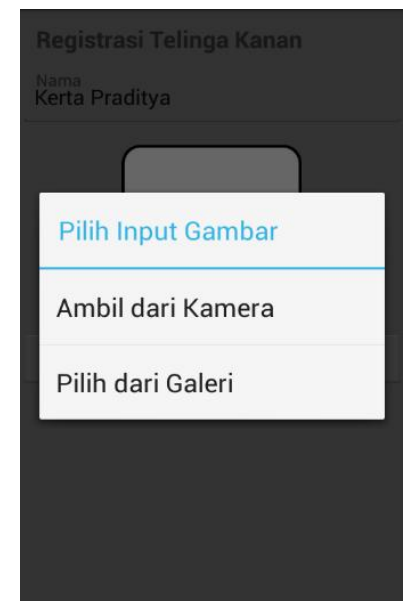

Fig 6: Select Image

Fig 6 is the display after the camera button on Fig. 8 selected. There are two optionsto input the image, that is through the camera or select from Android smartphone gallery.

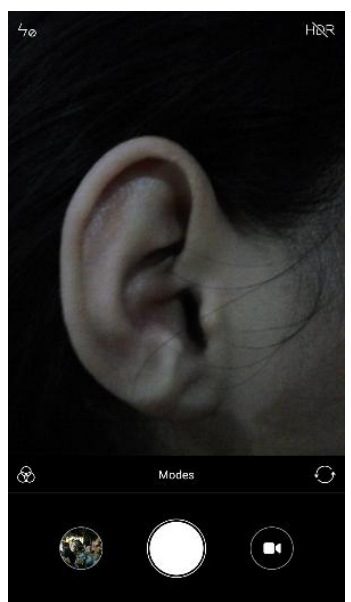

Fig 7: Image captured through Camera
Fig 7 is an image capture view through Android smartphone camera. This view adjusts to the camera view of the smartphone used.

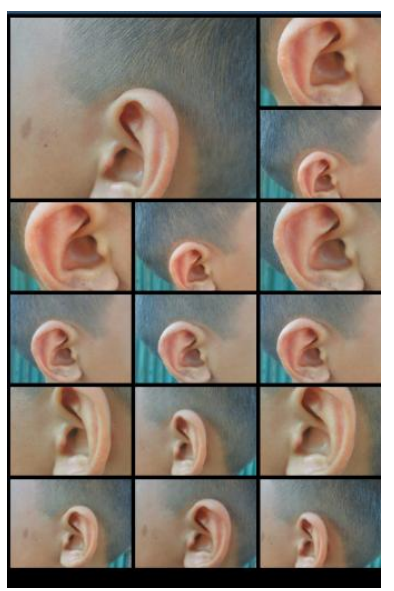

Fig 8: Image captured through Gallery

Fig 8 is the view of image captured through the gallery. This display will show all the images contained on the Android smartphone used.

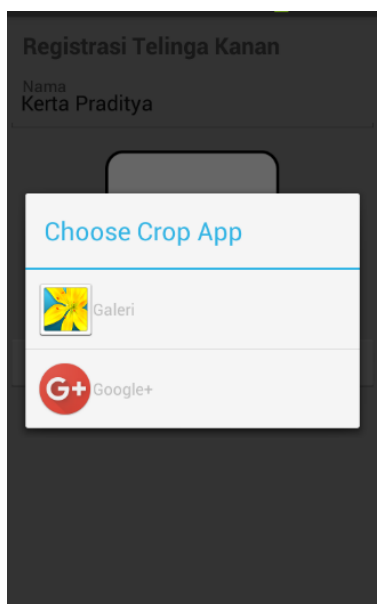

Fig 9: Choose Crop App

Fig 9 is an application selection display used to perform the cropping process. This view appears after the image is selected, whether the image is selected through the camera or gallery. This view adjusts to applications that can be used on Android smartphone used.

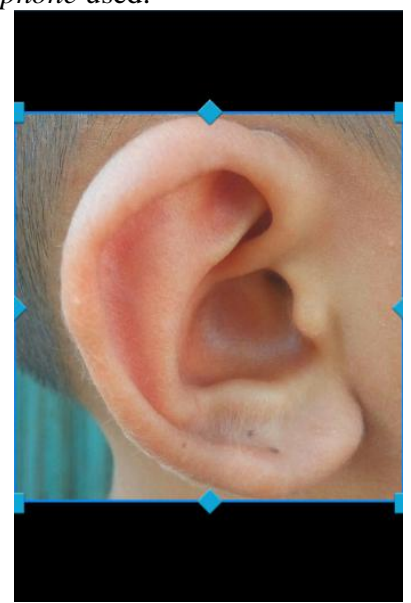




\section{Fig 10: Cropping}

Fig 10 is a cropping process display. The cropping process is done to get the desired ear part.

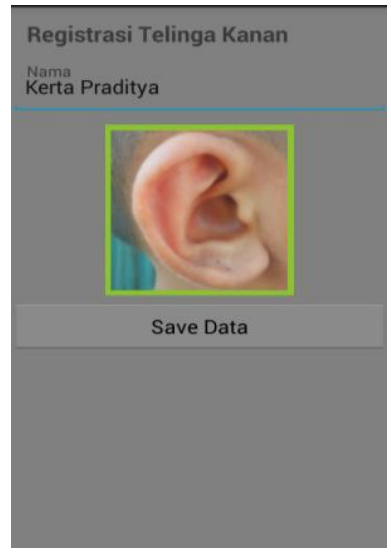

Fig 11: Registration feature

Fig 11 is the display after the cropping process done, after input the data completely then press the Save Data button. The next process is the registration of the left ear. The process of registering the left ear is the same as the right ear (from Fig 8 to Fig 12).

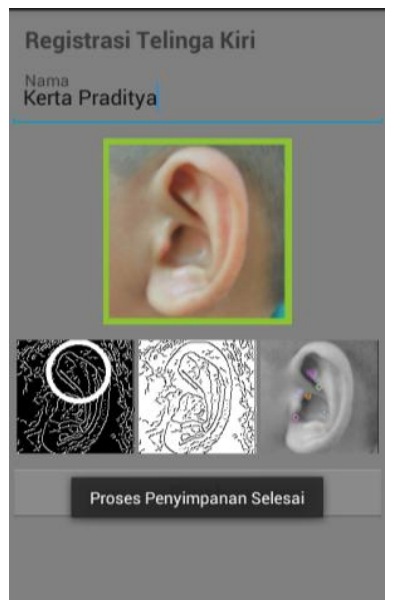

Fig 12 Registration Completed

Fig 12 shows the registration process of the right and left ear is done successfully.

\subsection{Recognition Features}

Recognition feature of the Application of Android-based Ear Biometrics Identification is a feature to perform the ear recognition process. The ear used as test data is one ear (right or left ear).

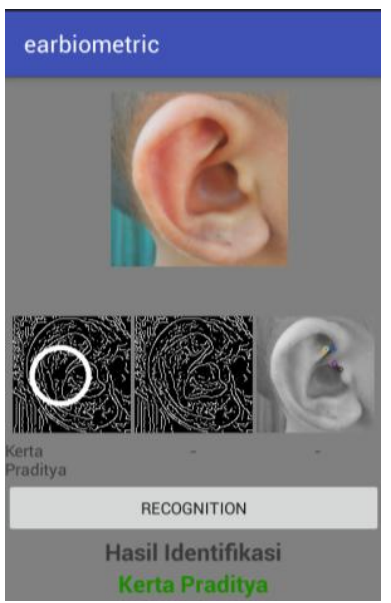

Fig 13: Recognition feature

Fig. 13 shows the result of identification after the RECOGNITION button is selected. This picture shows that the ear was successfully recognized on behalf of Kerta Praditya, of the three methods used that can recognize is the Hough Transform Method.

\section{RESULT OF APPLICATION TEST}

Testing on Application of Android-based Ear Biometrics Identification is done by using the ear image of the population in Bali both women and men with the age range of 1 to 70 years. Here is the ear data used as test data in this study.

Table 3. Test data

\begin{tabular}{|c|c|c|}
\hline \multirow{2}{*}{ Age Range } & \multicolumn{2}{|c|}{ Number of data } \\
\cline { 2 - 3 } & Left ear & Right ear \\
\hline $1-10$ & 20 & 20 \\
\hline $11-20$ & 20 & 20 \\
\hline $21-30$ & 20 & 20 \\
\hline $31-40$ & 20 & 20 \\
\hline $41-50$ & 20 & 20 \\
\hline $51-60$ & 20 & 20 \\
\hline $61-70$ & 20 & 20 \\
\hline Total & 140 & 140 \\
\hline
\end{tabular}

Each ear was taken as a sample image with a size of $4608 \times 3456$ pixels taken with a distance of $40 \mathrm{~cm}$ and then converted into 150x150 pixel image.

Table 4. Right Ear Success Rate Test Results

\begin{tabular}{|c|r|r|r|}
\hline Age Range & \multicolumn{1}{|c|}{ Canny } & \multicolumn{1}{|c|}{ Hough } & \multicolumn{1}{c|}{ ORB } \\
\hline $1-10$ & 12 & 14 & 10 \\
\hline $11-20$ & 12 & 10 & 10 \\
\hline $21-30$ & 14 & 10 & 10 \\
\hline $31-40$ & 12 & 12 & 10 \\
\hline $41-50$ & 14 & 12 & 12 \\
\hline $51-60$ & 12 & 10 & 10 \\
\hline $61-70$ & 14 & 12 & 10 \\
\hline Success Rate & 64 & 57 & 51 \\
\hline
\end{tabular}

Table 4 shows the results of application test on the right ear obtained the different percentages of the successful 
recognition; using Canny method obtained $64 \%$ of success, with Hough method of $57 \%$ and ORB method of $51 \%$.

\section{Table 5. Right Ear FAR Test Results}

\begin{tabular}{|c|r|r|r|}
\hline Age Range & \multicolumn{1}{|c|}{ Canny } & \multicolumn{1}{|c|}{ Hough } & \multicolumn{1}{c|}{ ORB } \\
\hline $1-10$ & 4 & 4 & 0 \\
\hline $11-20$ & 0 & 2 & 0 \\
\hline $21-30$ & 6 & 10 & 0 \\
\hline $31-40$ & 0 & 0 & 0 \\
\hline $41-50$ & 0 & 2 & 0 \\
\hline $51-60$ & 4 & 6 & 0 \\
\hline $61-70$ & 4 & 6 & 0 \\
\hline FAR & 12 & 21 & 0 \\
\hline
\end{tabular}

Table 5 shows the results of application test on the right ear obtained the different percentages of the FAR recognition; using Canny method obtained $12 \%$ of success, with Hough method of $21 \%$ and ORB method of $0 \%$.

Table 6. Left Ear Succes Rate Test Results

\begin{tabular}{|c|r|r|r|}
\hline Age Range & \multicolumn{1}{|c|}{ Canny } & \multicolumn{1}{|c|}{ Hough } & \multicolumn{1}{c|}{ ORB } \\
\hline $1-10$ & 12 & 14 & 10 \\
\hline $11-20$ & 12 & 10 & 10 \\
\hline $21-30$ & 14 & 10 & 10 \\
\hline $31-40$ & 12 & 12 & 10 \\
\hline $41-50$ & 14 & 12 & 12 \\
\hline $51-60$ & 12 & 10 & 10 \\
\hline $61-70$ & 14 & 12 & 10 \\
\hline Success Rate & 64 & 57 & 51 \\
\hline
\end{tabular}

Table 6 shows the results of application test on the left ear obtained the different percentages of the successful recognition; using Canny method obtained $64 \%$ of success, with Hough method of $57 \%$ and ORB method of $51 \%$.

Table 7. Left Ear FAR Test Results

\begin{tabular}{|c|r|r|r|}
\hline Age Range & \multicolumn{1}{|c|}{ Canny } & Hough & ORB \\
\hline $1-10$ & 6 & 4 & 0 \\
\hline $11-20$ & 0 & 2 & 0 \\
\hline $21-30$ & 4 & 6 & 0 \\
\hline $31-40$ & 0 & 0 & 0 \\
\hline $41-50$ & 0 & 2 & 0 \\
\hline $51-60$ & 4 & 6 & 0 \\
\hline $61-70$ & 4 & 6 & 0 \\
\hline FAR & 12 & 18 & 0 \\
\hline
\end{tabular}

Table 7 shows the results of application test on the left ear obtained the different percentages of the FAR recognition; using Canny method obtained $12 \%$ of success, with Hough method of $18 \%$ and ORB method of $0 \%$.

Table 8. Overall Test Results

\begin{tabular}{|c|c|c|}
\hline Age Range & Left ear & Right ear \\
\hline $1-10$ & 12 & 12 \\
\hline $11-20$ & 10 & 10 \\
\hline $21-30$ & 8 & 8 \\
\hline $31-40$ & 12 & 12 \\
\hline $41-50$ & 12 & 12 \\
\hline
\end{tabular}

\begin{tabular}{|c|c|c|}
\hline $51-60$ & 10 & 10 \\
\hline $61-70$ & 12 & 12 \\
\hline Success Rate & 54 & 54 \\
\hline
\end{tabular}

Table 8 shows the results test of the successful recognition to the three methods used, in which the minimum identified must recognizable by the two methods, so there are no FAR result. Canny Edge Detection is a method with a success rate of $64 \%$ for the right ear and left ear. Success rate for Hough Transform achieved a 54\% for the right ear and left ear, and for the ORB Method only managed to recognize $51 \%$. Canny Edge Detection used to detect the shape of the ear by looking for edges, this method have the highest accuracy. This shows that the shape of each person's ear is different. Identification by using Hough Transform is lower than Canny Edge Detection whereas Hough Trasform has different center point for different image. ORB method the accuracy is very low because this method looks for keypoint based on texture. Texture is very difficult to detect through images. ORB method most influence by light intensity. The different of light intensity will make different result of image identification. The recognition between right and left images has no significant difference. The different of age range due to image retrieval for databases with test data taken at the same age had no influences. The combination result of Canny, Hough, and ORB Detection for the right and left ear is $54 \%$. The detection result is lower than Canny because the ear is recognized when the two methods manage to recognize the same person. The ear will not be recognized if it only recognizes one of the methods.

\section{CONCLUSIONS}

Based on the research conducted in the recognition of ear biometrics with the three methods; Canny Edge Detection, Hough Transform, and ORB, it can be concluded that the success rate using Hough Transform is higher than using Canny and ORB. The result of combining the three methods resulted success accuracy of 54\%. The results of recognition of differences in the right and left ear have no major effect. The failure rate is mostly due to improper image capture, such as improper image capture positioning, lighting, and hair that is sometimes considered as feature. The differenf of age range have no influence on the results of identification, future testing can be done at the age of the sample has been increased.

\section{ACKNOWLEDGMENTS}

Thank you Udayana University for the support in this study, especially thanks to the Department of Information Technology, Faculty of Engineering, Udayana University.

\section{REFERENCES}

[1] M. Lourde and D. Khosla, "Fingerprint Identification in Biometric SecuritySystems," Int. J. Comput. Electr. Eng., vol. 2, no. 5, p. 852, 2010.

[2] D. Rusjayanthi, "Deviasi , dan K-Means Clustering," vol. 4, no. 2, pp. 265-276, 2013.

[3] Darma Putra, Sistem Biometrika: Konsep Dasar, Teknik Analisis Citra, dan Tahapan Membangun Aplikasi Sistem Biometrika. Yogyakarta: Andi, 2009.

[4] I. N. Piarsa and R. Hisamuddin, "Sistem Verifikasi Online Menggunakan Biometrika Wajah,” vol. 9, no. 1, 2010.

[5] S. S. Mudholkar, P. M. Shende, and M. V Sarode, 
"Biometrics Authentication Technique for Intrusion Detection Systems Using Fingerprint Recognition," Int. J. Comput. Sci. Eng. Inf. Technol., vol. 2, no. 1, pp. 5765, 2012.

[6] N. B. Boodoo and R. K. Subramanian, "Robust multibiometric recognition using face and ear images," Int. J. Comput. Sci. Inf. Secur., vol. 6, no. 2, pp. 164-169, 2009.

[7] D. Suryadi, R. Hidayat, and H. A. Nugroho, "Pengembangan Sistem Identifikasi Multimodal Dengan Mengunakan Wajah Dan Telinga," vol. 2014, no. Sentika, 2014.

[8] Ernastuti, "Implementasi Metode Hough Dan Jarak Mahalanobis Pada Sistem Biometrik Pengenalan Telinga Dengan Menggunakan Library Open CV,” 2012.

[9] S. Gunawan, Biologi untuk SMA/MA Kelas XI. Jakarta: Grasindo, 2007.

[10] Risky, Big Book Ilmu Pengetahuan Alam SD Kelas 4,5, \& 6. Jakarta: Cmedia Imprint Kawan Pustaka, 2015.

[11] L. Widya, Buku Ajar Biologi Dasar dan Biologi Perkembangan (Kebidanan). Yogyakarta: Nuha Medika, 2015.

[12] M. Choraś, "Ear Biometrics Based on Geometrical Feature Extraction," Electron. Lett. Comput. Vis. Image Anal., vol. 5, no. 3, pp. 84-95, 2005.
[13] Iannarelli, Ear Identification, Forensic I. California: Paramont Publishing Company, 1989.

[14] H. Yang, N. Zhang, Q. Zeng, Q. Yu, S. Ke, and X. Li, "HPLC Method for the Simultaneous Determination of Ten Annonaceous Acetogenins after Supercritical Fluid CO2 Extraction.," Int. J. Biomed. Sci., vol. 6, no. 3, pp. 202-7, 2010.

[15] K. H. Pun and Y. S. Moon, "Recent advances in ear biometrics," Proc.of Sixth IEEE Int'l Conf. Autom. Face GestureRecognition, pp. 164-169, 2004.

[16] J. Canny, A Computational Approach to Edge Detection. 1986.

[17] Darma Putra, Pengolahan Citra Digital. Yogyakarta: Andi, 2010.

[18] A. Mcandrew, "An Introduction to Digital Image Processing with Matlab Notes for SCM2511 Image Processing 1 Semester 1, 2004,” 2004.

[19] E. Rublee, V. Rabaud, K. Konolige, and G. Bradski, "ORB: An efficient alternative to SIFT or SURF," Proc. IEEE Int. Conf. Comput. Vis., no. November 2011, pp. 2564-2571, 2011.

[20] N. K. A. Wirdiani and A. A. K. Oka Sudana, "Medicinal plant recognition of leaf shape using Localized Arc Pattern Method," Int. J. Eng. Technol., vol. 8, no. 4, pp. 1847-1854, 2016. 